\title{
Determinantes asociados a depresión crónica e incidente en adultos mayores mexicanos
}

\author{
A.L. Sosa Ortiz', C.I. Astudillo García ${ }^{2}$ y G.I. Acosta Castillo ${ }^{1}$ \\ ${ }^{1}$ Laboratorio de Demencias, Instituto Nacional de Neurología y Neurocirugía; ${ }^{2}$ Servicios de Atención Psiquiátrica, Secretaría de Salud, Ciudad de \\ México, México
}

\begin{abstract}
Resumen
Objetivo: Describir la asociación de determinantes sociodemográficos, del entorno, psicosociales y de salud en adultos mayores (AM) mexicanos con depresión crónica e incidente, en un estudio poblacional de seguimiento a 3 años. Método: Se analizaron los datos de un estudio de cohorte de 1512 mexicanos de 65 años y más. Mediante un modelo de regresión multinomial se analizaron las asociaciones de los determinantes de interés, con los grupos de depresión crónica e incidente, tomando como referencia al grupo sin depresión. Se identifican los perfiles de riesgo por sexo, con los determinantes evaluados individualmente y en combinaciones. Resultados: El $43.1 \%$ de la muestra no presentó depresión a lo largo del seguimiento, el $41.9 \%$ presentó depresión crónica, y el 15\% mostró depresión incidente. La depresión crónica tuvo un mayor número de asociaciones en comparación con la depresión incidente; la última estuvo relacionada con condiciones de pobreza y soledad. Los perfiles de riesgo para pertenecer a los grupos de depresión, por sexo, mostraron notables diferencias. Conclusión: La depresión crónica e incidente en el AM son fenómenos distintos. La evaluación de la contribución de los determinantes permitirá identificar condiciones asociadas a la depresión crónica e incidente, así como el efecto diferencial por sexo, todo lo cual permitirá identificar grupos y factores de riesgo sobre los cuales se puedan implementar intervenciones.
\end{abstract}

PALABRAS CLAVE: Adultos mayores. Depresión. Determinantes sociales de la salud.

\begin{abstract}
Objective: To describe the association among socio-demographic, environmental, psychosocial and health determinants in Mexican elders with chronic and incident depression from a 3-year follow-up study. Methods: Data from a cohort study of 1512 Mexicans, 65 and more years old was analyzed. We used a multinomial regression model in order to analyze the associations between determinants of interest with chronic and incident depression conditions, taking as reference a group without depression. In addition, the risk profiles were identified and stratified by sex; and finally, the determinant's were estimated individually and combined. Results: $43.1 \%$ of the whole sample did not present depression during the follow-up, $41.9 \%$ had chronic depression and $15 \%$ incident depression. Chronic depression had more associations with determinants, than incident depression; the latter was associated with poverty and loneliness. The risk profiles for the three depression's conditions stratified by sex, showed notorious differences. Conclusion: Chronic and incident depressions in the elderly are distinct phenomena, the evaluation of the determinant's contribution to these entities, will allow to identify associated conditions to chronic and incident depression, and the sex differential effect, which will contribute to identify populations and risk factors, to implement objective interventions.
\end{abstract}

KEY WORDS: Older adults. Depression. Social determinants of health.

\author{
Correspondencia: \\ A.L. Sosa Ortiz \\ Insurgentes Sur, 3877 \\ Col. La Fama, C.P. 14269 , \\ Ciudad de México, México \\ E-mail: drasosa@ hotmail.com
}

Fecha de recepción: 07-08-2017

Fecha de aceptación: 07-09-2017

DOI://dx.doi.org/10.24875/GMM.M17000011
Gac Med Mex. 2017;153;Sup 2:S102-S118

Contents available at PubMed

www.anmm.org.mx 


\section{Introducción}

El envejecimiento es un proceso en el cual se suman carácteristicas biológicas y psicosociales a lo largo de la vida, cuyo resultado es la generación de una población muy heterogénea de personas mayores, que comparten la característica de ser más susceptibles a enfermar ${ }^{1}$. En los últimos 50 años, el envejecimiento poblacional en todo el mundo está ocurriendo de manera vertiginosa, y las estimaciones indican que este se continuará presentando, y que se dará de manera más acelerada. En las próximas décadas, la proporción de adultos mayores (AM) en el mundo se duplicará, pasando de representar el $12 \%$ en 2015 al 25\% para el 2050; dicho cambio poblacional se acompañará también de una transición epidemiológica caracterizada por la preponderancia de enfermedades crónicas, entre las que ocupan un lugar importante las enfermedades mentales. Cerca del $20 \%$ de los AM padecen algún problema de salud mental $(S M)^{2,3}$, y estos trastornos representan el $28.0 \%$ del total de años de vida ajustados por discapacidad para este grupo de edad 4 .

La depresión es el trastorno mental más frecuente en el AM. Su origen y manifestaciones tienen componentes biopsicosociales que interactúan de manera compleja'. Se ha observado que la depresión está asociada, además de al sufrimiento que ocasiona, a deterioro cognitivo, funcional y de la calidad de vida, aumento en el riesgo de morbilidad y mortalidad por enfermedades médicas, y mayor tasa de utilización de servicios de salud y costos, además de que el impacto de la comorbilidad médica parece ser bidireccional, pues se asocia con un peor pronóstico de las enfermedades médicas, que a su vez afecta de manera negativa el curso de la depresión ${ }^{5}$.

Estudios que han evaluado el curso longitudinal de la depresión en los AM señalan que hay variaciones importantes en sus trayectorias ${ }^{6-10}$; mientras en algunos remiten, en otros persisten, y en otros más los síntomas se incrementan o decrementan a lo largo del tiempo. Las condiciones que se asocian con una u otra trayectoria suelen ser sociodemográficas y de salud $^{11}$, dando por resultado una amplia heterogeneidad a lo largo de su evolución.

Un abordaje que nos puede ayudar a analizar estas diferencias en el curso de la depresión es la perspectiva del curso de vida, la cual muestra cómo las exposiciones de riesgo en diferentes etapas pueden afectar la SM o predisponer al desarrollo y el mantenimiento de alguna alteración mental años o décadas después ${ }^{12}$.

La SM y el bienestar emocional son condiciones importantes en todas las etapas de la vida. Ha sido insistentememte señalado que, sin importar la edad, «no hay salud $\sin S M{ }^{4}$, siendo la depresión del AM un problema de salud pública por su alta prevalencia e importantes consecuencias, por lo que es imperativo identificar sus factores de riesgo y determinantes para diseñar intervenciones tempranas, preventivas y terapéuticas que incidan sobre los riesgos potencialmente modificables. Por ello, esta investigación tiene como objetivo describir la asociación de determinantes sociodemográficos, del entorno, psicosociales y de salud en AM mexicanos con depresión crónica e incidente, en un estudio de seguimiento a 3 años.

\section{Métodos}

El presente trabajo consiste en el análisis del seguimiento a 3 años de 1512 participantes, de un universo de 2003 adultos mexicanos de 65 años o más, estudiados con los protocolos del Grupo de Investigación en Demencias 10/66 (GID 10/66) (Fig. 1).

Para el presente reporte solo se consideraron sujetos que completaron las evaluaciones inicial y de seguimiento, con o sin depresión en cada una de ellas, de los cuales fueron excluidos los AM que habiendo presentado depresión en la evaluación inicial no estuvo presente en el seguimiento, es decir, los que se recuperaron, debido a que consideramos que corresponden a un grupo particular cuyo abordaje e interpretación están fuera del objetivo de este trabajo. Los detalles de la selección, de las áreas de estudio, de la evaluación y del seguimiento de los sujetos se han documentado en reportes específicos ${ }^{13,14}$. En términos generales, se trata de residentes de áreas delimitadas en zonas urbanas o rurales, de ingreso económico medio y bajo, en México. Para la selección de la muestra, en el área urbana se incluyeron cuatro áreas geoestadísticas básicas de la delegación Tlalpan, ubicada al sur de Ciudad de México; para el área rural, la zona de reclutamiento quedó conformada por los límites territoriales de 10 pueblos de los municipios de Huitzilac y Tepoztlán, en el estado de Morelos. El reclutamiento y la evaluación basal fueron realizados mediante un censo puerta a puerta entre 2005 y 2007. Tres años después (2008-2010), los sujetos evaluados fueron recontactados para la evaluación de seguimiento. En ambas evaluaciones (basal $y$ de seguimiento) se aplicaron los mismos 


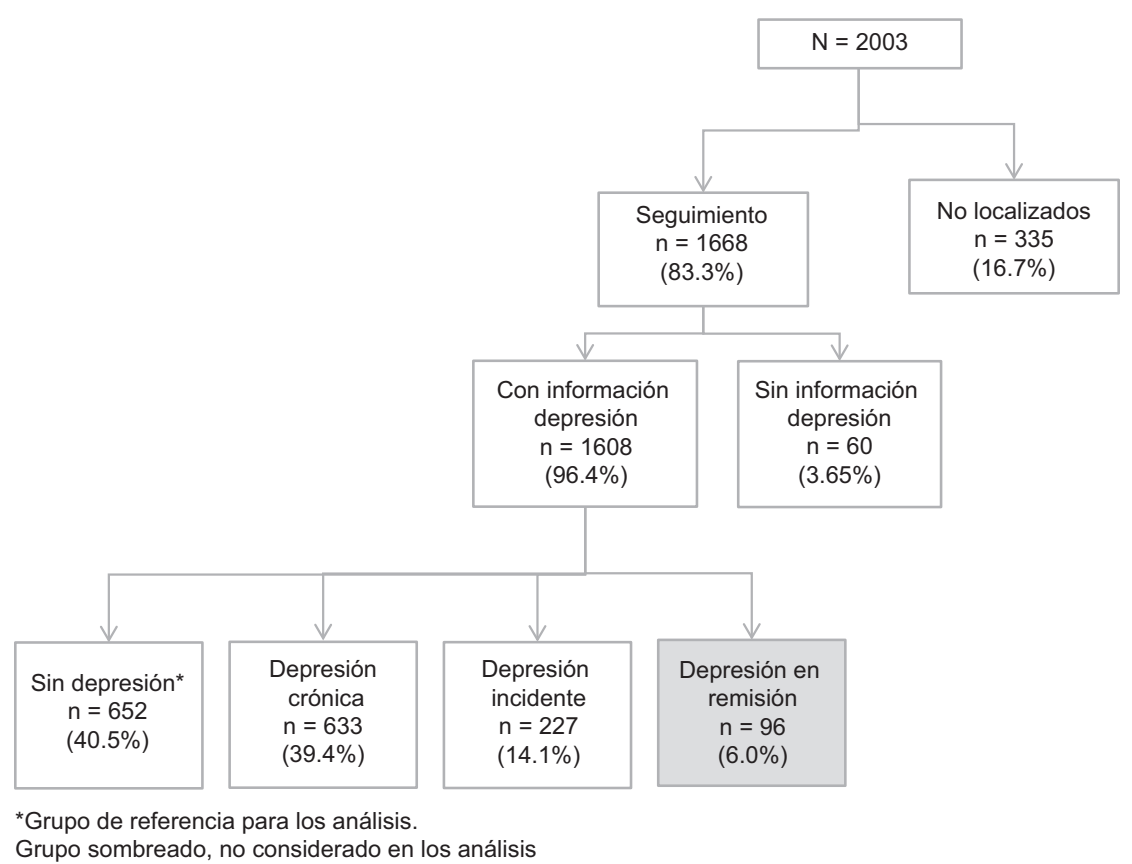

Figura 1. Flujograma de la muestra analítica.

instrumentos, los cuales fueron: 1) cuestionario del hogar, 2) evaluación cognitiva, 3) inventario del estado mental geriátrico, 4) cuestionario sociodemográfico y de factores de riesgo, y 5) exploración neurológica y física general. Además de la evaluación a los participantes, se aplicó otra entrevista estructurada a un informante/cuidador del AM.

Todos los AM estudiados aceptaron participar en el estudio mediante la firma de un consentimiento informado, o la impresión de su huella digital en presencia de un testigo en el caso de sujetos que no podían leer o escribir. El protocolo fue sometido y autorizado por los comités de bioética e investigación del Instituto Nacional de Neurología y Neurocirugía y del King's College de Londres, Inglaterra.

\section{Mediciones}

\section{Variable dependiente}

La variable dependiente fue la depresión. Se consideraron casos de depresión cuando los AM cumplían con uno o más de los siguientes diagnósticos: 1) síndrome depresivo, identificado mediante el inventario del estado mental geriátrico GMS-AGECAT (Geriatric Mental State-Automatic Geriatric Examination for Computer-Assisted Taxonomy) ${ }^{15}$; 2) episodio depresivo, de acuerdo con los criterios de la Clasificación Internacional de Enfermedades (10. ${ }^{a}$ edición); 3) depresión mayor, de acuerdo con los criterios del Manual Diagnóstico y Estadístico de los Trastornos Mentales (DSM-IV); y 4) depresión subsindromática, derivada de la escala EURO-D ${ }^{16}$. La utilización de estos criterios permitió cubrir un espectro amplio de los trastornos depresivos, que van desde sintomatología depresiva clínicamente significativa hasta una aproximación sindromática y de enfermedades específicas.

Según la presencia o ausencia de depresión en ambas mediciones (basal y seguimiento) se formaron tres grupos, constituidos por sujetos: 1) sin depresión: donde se incluyeron aquellos que no resultaron casos en ninguna de las dos mediciones; 2) con depresión crónica: para aquellos que fueron identificados como casos de depresión en ambas mediciones; y 3) con depresión incidente: aquellos que no se identificaron como casos de depresión en la evaluación basal, pero sí en el seguimiento.

\section{Variables de exposición}

- Sociodemográficas: se consideraron el sexo, el grupo de edad al inicio del estudio (clasificados en cuatro intervalos: $65-69,70-74,75-79$ y 80 y más años), la escolaridad según el último grado acreditado (categorizado en cuatro grupos: escolaridad nula, primaria incompleta, primaria completa, secundaria o superior), el estado civil 
(casados/unión libre, solteros, viudos, divorciados) y la ocupación reportada en la evaluación inicial (retirado, trabajo de tiempo completo o medio tiempo, en búsqueda de empleo y actividades del hogar).

- Entorno y condición socioeconómica: se consideró el área de residencia como urbana o rural, clasificada considerando el tamaño de población, localización y principal actividad económica.

- La inseguridad alimentaria, evaluada a través de la respuesta afirmativa a la pregunta «alguna vez se ha quedado con hambre porque no hay suficiente comida», que se considera un indicador de pobreza extrema.

- Activos o servicios en el domicilio, variable creada mediante la suma de siete indicadores relacionados con tener servicios y bienes en el domicilio (televisión, refrigerador, agua potable, electricidad, teléfono, drenaje y baño con regadera), los cuales fueron utilizados como un referente de la condición socioeconómica del domicilio; esta información sirvió para crear una variable dicotómica clasificando a los hogares como igual o menos de 3 activos o servicios y 4 o más.

- Factores psicosociales: en este rubro consideramos la frecuencia de contacto con familiares, amigos y vecinos. Esta información se recolectó de manera separada para familiares, amigos y vecinos, y cada una de ellas se codificó en tres categorías. Para el contacto con familiares fueron: diario, al menos una vez a la semana y menos de una vez a la semana. Para la frecuencia de contacto con amigos y vecinos: nunca, al menos una vez al mes, al menos una vez a la semana. También se consideró la frecuencia de asistencia a actividades religiosas, grupos sociales 0 actividades en la comunidad. Esta información se evaluó de manera separada y fue codificada como: no asiste y asiste regular $u$ ocasionalmente. Vivir solo se codificó según la información obtenida de la composición del hogar, y se diferenciaron los sujetos que tenían corresidentes y los que vivían solos. La soledad se determinó según si el participante reportaba sentirse solo o no, como respuesta directa a la pregunta contenida en Inventario del Estado Mental Geriátrico $0^{15}$. La satisfacción por la vida se evaluó preguntando "cómo describiría su satisfacción por la vida», recodificando las tres respuestas originales (buena, regular y mala) en dos categorías: satisfecho, para la primera opción, e insatisfecho, para el resto. Para evaluar la felicidad se preguntó al sujeto qué tan feliz y qué tan contento se sentía, y también se recodificaron las respuestas originales (muy feliz, feliz, no muy feliz e infeliz) en dos categorías: feliz para las primeras dos opciones y poco o nada feliz para las dos últimas.

- Indicadores de salud: se construyó una variable sobre el estilo de vida, articulando el autorreporte de actividad física, consumo riesgoso de alcohol (bebedores fuertes) y tabaquismo. La actividad física se clasificó como nula, moderada o alta. Para el consumo de alcohol, se clasificó como bebedores fuertes a los sujetos que consumían 21 o más tragos a la semana para los hombres y 140 más para las mujeres ${ }^{17}$. En relación al tabaquismo, se investigó sobre uso de tabaco presente o pasado, la cantidad y el tiempo de consumo; para el presente reporte se utilizó la medida de paquetes por año, considerando como tabaquismo cuando se estimaba que el participante había consumido 20 o más paquetes al año ${ }^{17}$. En cuanto a la autopercepción del estado de salud, para documentarla se preguntó "cómo calificaría la calidad de su salud en el último mes", recodificando las cinco respuestas originales (muy buena, buena, regular, mala y muy mala) en tres categorías: buena para las dos primeras, regular y mala (para las dos últimas).

- Alteraciones cognitivas en el desarrollo: esta variable fue creada a partir del reporte del informante/cuidador o del evaluador considerando si el sujeto ha presentado alteraciones de las funciones cognitivas y si estas podrían ser atribuidas a un problema propio del desarrollo en etapas tempranas, y no a un problema de salud adquirido en la etapa adulta. En la categoría de deterioro cognitivo se incluyeron los casos identificados como deterioro cognitivo leve o demencia. El deterioro cognitivo leve lo definimos de acuerdo con los criterios de la Clínica Mayo para deterioro cognitivo leve amnésico, operacionalizados mediante un algoritmo desarrollado por el GID 10/66 18 ; la demencia, según la presencia de síndrome demencial, definida a partir de los criterios del DSM-IV y del algoritmo también desarrollado por el GID 10/66, mismos que han sido validados en población de ingreso económico medio 0 bajo ${ }^{19}$ y en estudios poblacionales 
teniendo el juicio clínico como método de referencia ${ }^{20}$.

- Antecedentes personales de depresión: documentada mediante el reporte de historia personal de depresión, vacío o tristeza que hubiera afectado sus actividades de la vida diaria, con duración de al menos 2 semanas.

- Número de enfermedades físicas limitantes: reportadas a partir de un listado de 11 condiciones autorreportadas como limitantes de las actividades diarias: 1) artritis o reumatismo; 2) problemas de la vista; 3) dificultades para escuchar o sordera; 4) tos frecuente o crónica; 5) dificultad para respirar o asma; 6) hipertensión arterial; 7) problemas cardiacos; 8 ) problemas del estómago 0 intestinales; 9) desmayos o pérdida momentánea de conocimiento; 10) parálisis, debilidad o pérdida de sensibilidad de una pierna o brazo; y 11) problemas de la piel. Con la suma de estos padecimientos se categorizó en la presencia de ninguna, una $o$ dos, y tres o más enfermedades que limitan físicamente ${ }^{13}$.

\section{Análisis}

Se describieron las características de la muestra estudiada por sexo y por grupos de acuerdo con su condición de depresión (sin depresión, depresión crónica y depresión incidente). Las variables de interés fueron agrupadas de la siguiente manera: a) sociodemográficas, b) del entorno, c) psicosociales y d) indicadores de salud. Posteriormente se ajustó un modelo de regresión multinomial para estimar la asociación entre las variables de interés. Derivados del modelo, se obtuvieron razones de momios (OR, odds ratio) con su correspondiente intervalo de confianza al $95 \%$ (IC 95\%). Para la selección de los modelos se consideraron los criterios de información Akaike y Bayesiano ${ }^{21}$, como indicadores de ajuste, así como la relevancia clínica de las variables. Estas estimaciones se replicaron para hombres y mujeres de manera independiente.

Posteriormente, se estimaron valores de probabilidad de pertenencia a cada uno de los grupos de depresión por sexo, así como la probabilidad de pertenencia a los grupos según las características de mayor magnitud de asociación; finalmente, la probabilidad de pertenencia a cada grupo de depresión adicionando las condiciones de mayor asociación y prevalencia. Dicha probabilidad se presenta en forma de porcentaje para facilitar su interpretación.
Para todos los análisis se consideró el diseño de la muestra utilizando la vivienda como variable de agrupamiento. Todos los análisis se realizaron en el programa estadístico Stata 14.2.

\section{Resultados}

De los 1512 participantes, 986 (65.2\%) eran mujeres, $652(43.1 \%)$ conformaron el grupo de no depresión (en ninguna de las mediciones), 633 (41.9\%) el de depresión crónica y 227 (15.0\%) el de depresión incidente. En cuanto a escolaridad, predominaron los grupos de baja escolaridad (primaria incompleta $44.4 \%$ y escolaridad nula $26.7 \%$ ). El $51.6 \%$ de la muestra eran casados y el $36.7 \%$ viudos. Esta distribución y el resto de las características sociodemográficas y condiciones de salud por sexo y grupo de depresión se muestran en la tabla 1.

En relación con la condición laboral, el 48.4\% se dedicaban a actividades del hogar y el $22.1 \%$ trabajaba al momento de la evaluación inicial. En cuanto a la condición socioeconómica, el $6.7 \%$ reportó sufrir inseguridad alimentaria y el $20.6 \%$ contar con tres o menos activos y servicios en su domicilio.

El $70.0 \%$ de los participantes declaró tener contacto diario con sus familiares, el $41.8 \%$ contacto frecuente con amigos y el $63.4 \%$ contacto frecuente con vecinos. El $51.2 \%$ reportó no tener nunca contacto con amigos. El 92.0\% reportó asistir regularmente a reuniones religiosas y el $25.3 \%$ a reuniones sociales 0 comunitarias.

El $10.3 \%$ de los participantes vivían solos y el $37.3 \%$ se sentían solos. El $46.4 \%$ respondieron que se sentían satisfechos con su vida, y el $83.0 \%$ sentirse felices. El $49.0 \%$ reportó sentirse en buen estado de salud.

El $69.6 \%$ presentó un estilo de vida mejorable (consumo riesgoso de alcohol, tabaco o nula actividad física), y el $17.7 \%$ antecedentes personales de depresión. Se identificó una prevalencia de deterioro cognitivo del $11.8 \%$. El $41.4 \%$ presentaban una o dos enfermedades, y el $17.8 \%$ tres o más enfermedades (en ambos casos enfermedades que ocasionaban limitación física).

A partir del análisis bivariado, entre las condiciones para las variables de interés evaluadas y el grupo de depresión (sin depresión, con depresión crónica o incidente), en la población estudiada se observaron diferencias estadísticamente significativas ( $p \leq 0.05)$ para la mayoría de ellas (penúltima columna de la tabla 1), no así para los grupos de edad, la zona de 


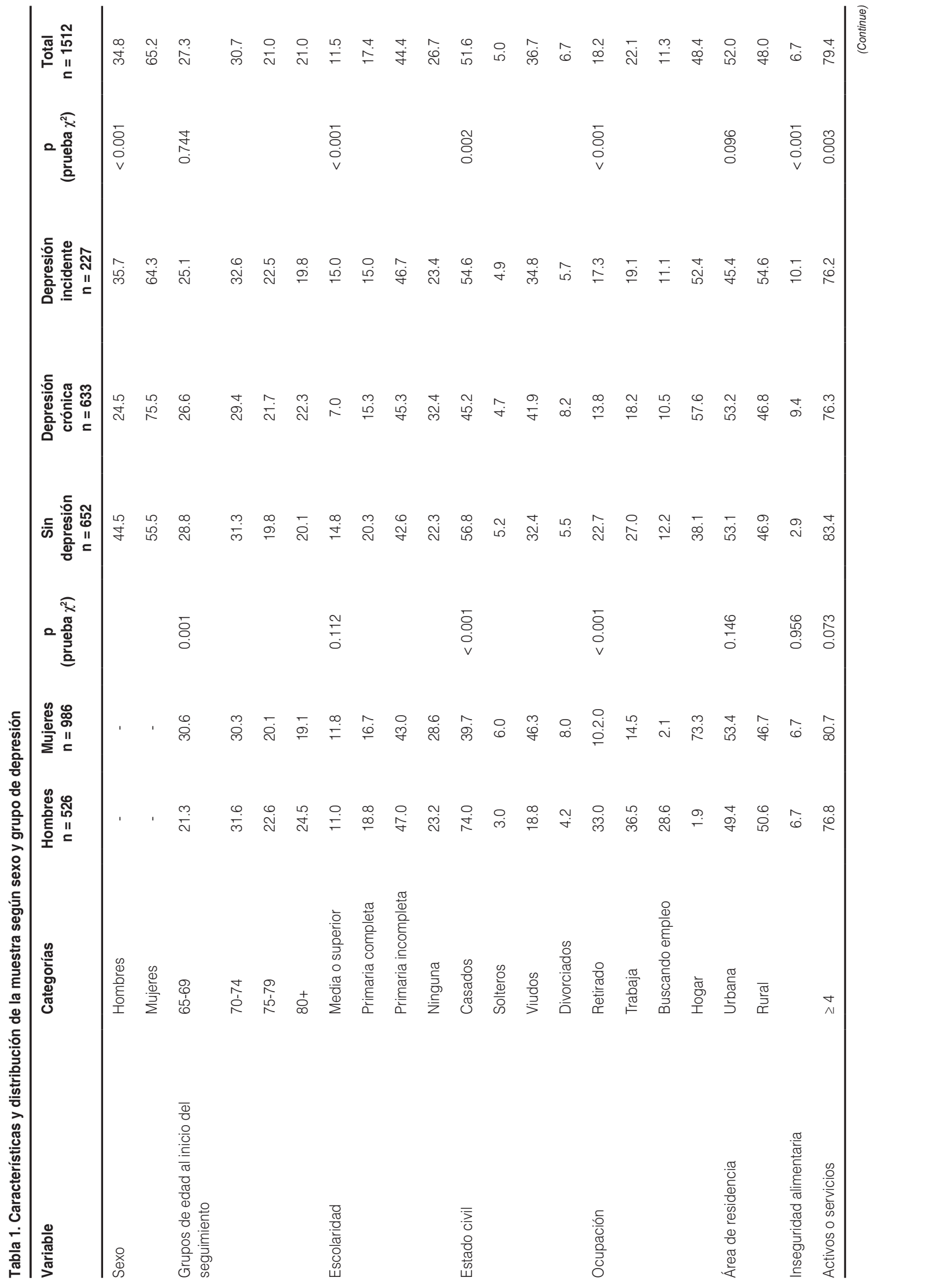




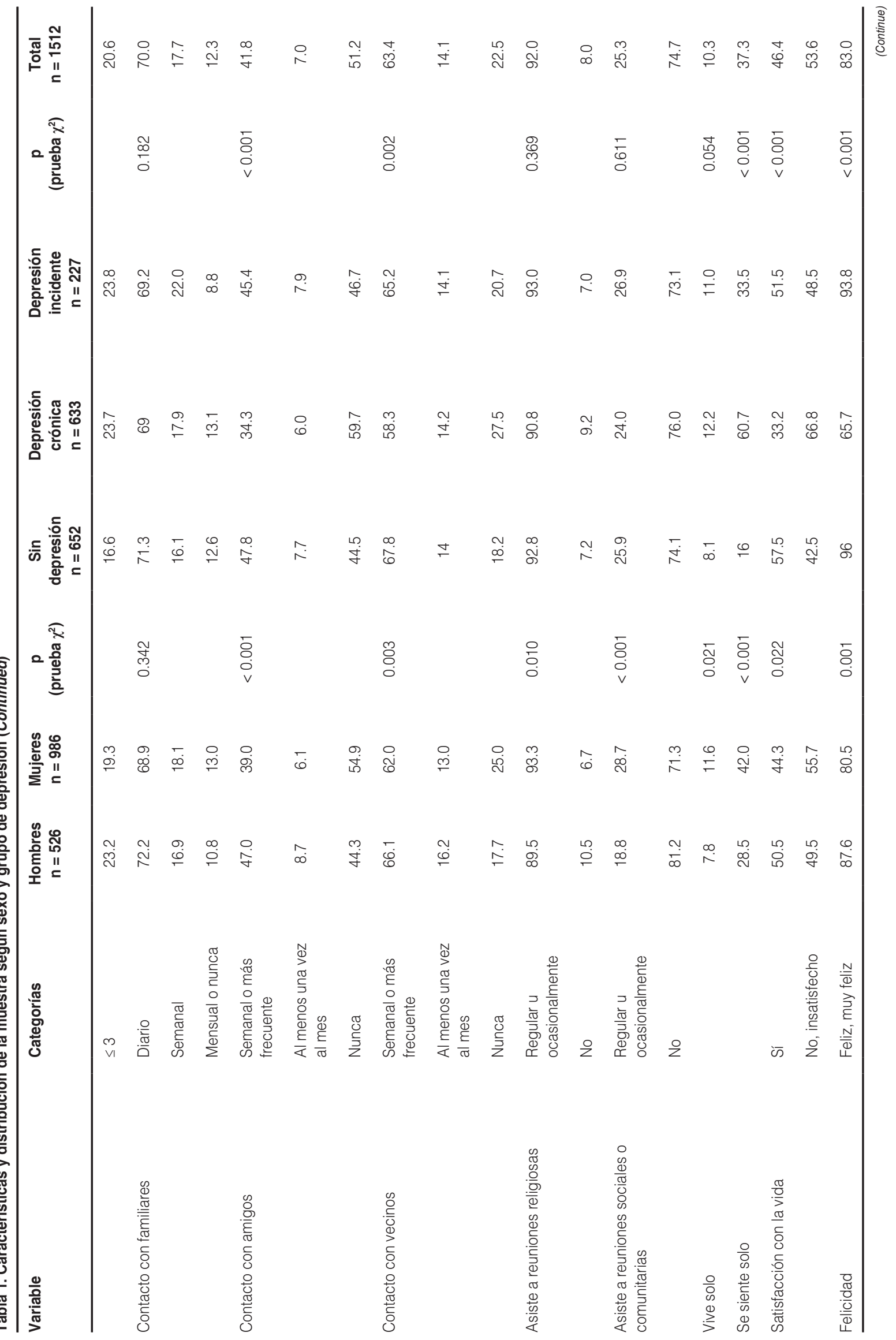



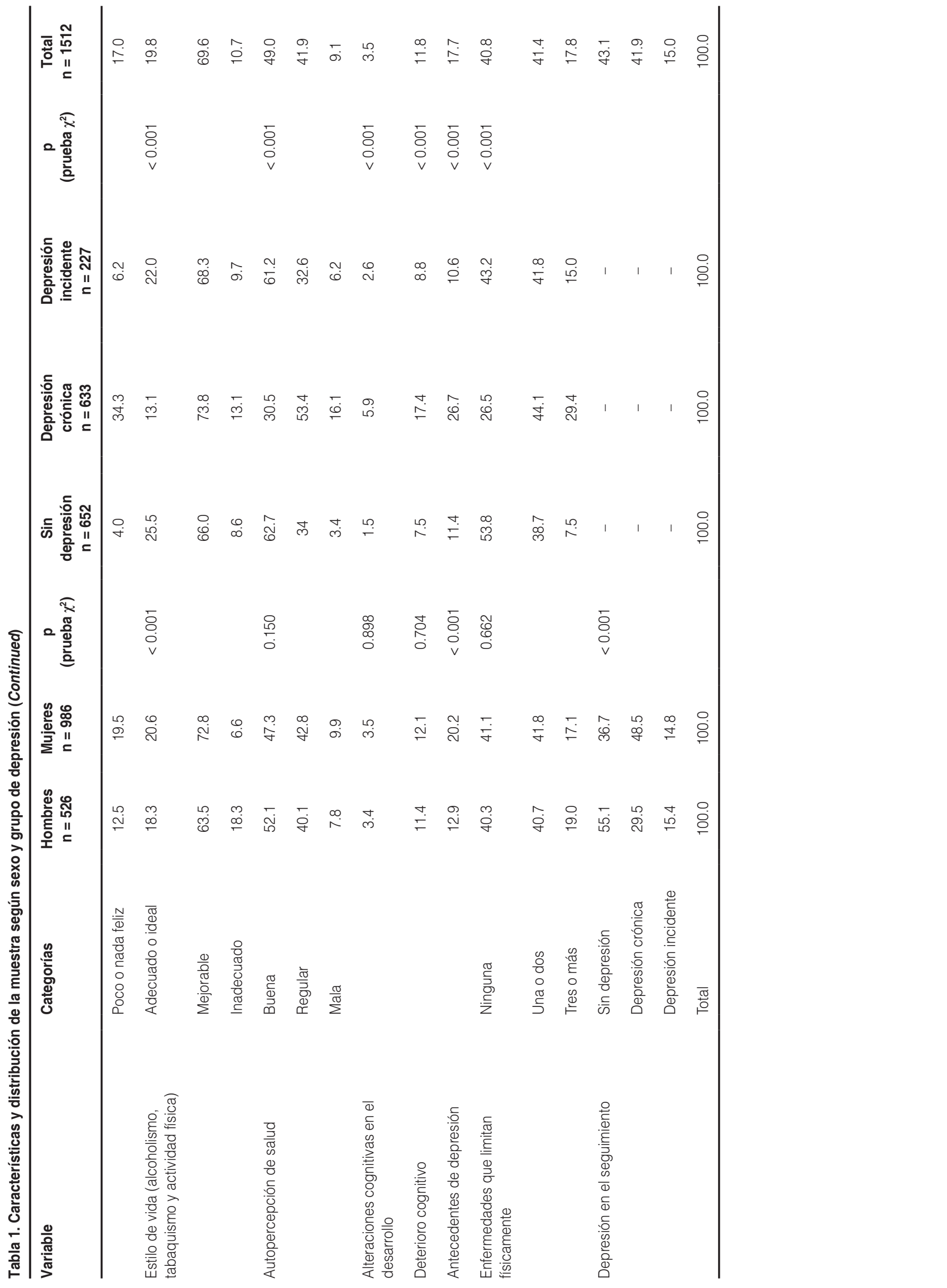
residencia, el contacto con familiares, asistir a reuniones religiosas, sociales o comunitarias, y vivir solo $(p>0.05)$.

Con respecto a las diferencias entre grupos, en el que se mantuvo libre de depresión destaca una mayor frecuencia de quienes trabajan $(27.0 \%)$, de aquellos sin inseguridad alimentaria $(97.1 \%)$, con más de cuatro bienes o servicios (83.4\%), sin sentimientos de soledad (84\%) y sin enfermedades discapacitantes (53.8\%). Para el grupo de depresión crónica, las condiciones más prevalentes fueron ser mujer $(75.5 \%)$, un porcentaje más alto de viudez (41.9\%), dedicarse a las labores del hogar $(57.6 \%)$, mayor porcentaje de personas que nunca tienen contacto con amigos $(59.7 \%)$ ni con vecinos $(27.5 \%)$, y más de la mitad reportaron sentirse solos $(60.7 \%)$ y estar insatisfechos $(66.8 \%)$. Otras características de este grupo son que el $34.3 \%$ reportaron ser poco o nada felices, el $13.1 \%$ tenían un estilo de vida no saludable, el $16.1 \%$ una autopercepción de mal estado de salud, el $5.9 \%$ alteraciones cognitivas en el desarrollo, el $17.4 \%$ deterioro cognitivo, el $26.7 \%$ antecedentes personales de depresión, y el $29.4 \%$ tres o más enfermedades que limitan físicamente. En el grupo de depresión incidente destaca una mayor frecuencia de residencia rural, con el $54.6 \%$, y un $10.1 \%$ de ellos sufren de inseguridad alimentaria.

\section{Modelo general}

En la tabla 2 se presentan las OR derivadas del modelo de regresión multinomial, que compara las condiciones sociodemográficas, del entorno, psicosociales y de salud de interés, para los grupos con depresión crónica e incidente, tomando como grupo de referencia el que se mantuvo libre de depresión en ambas mediciones. A continuación describimos las OR ajustadas (ORa) para cada grupo de depresión. En el caso de quienes presentaron depresión crónica, se observa que las condiciones de riesgo identificadas fueron ser mujer (ORa: 2.65; IC 95\%: 1.67- 4.21), haber cursado primaria incompleta (ORa: 1.98; IC 1.18-3.32), no tener escolaridad (ORa: 2.31; IC 95\%: 1.33-4.02), vivir en zona urbana (ORa: 1.68; IC 95\%: 1.21-2.33) y presentar inseguridad alimentaria (ORa 1.96; IC 95\%: 1.033.73); y entre las variables psicosociales, no tener contacto con amigos (ORa: 1.40; IC 95\%: 1.04-1.90), sentirse solo (ORa: 4.63; IC 95\%: 3.34-6.44), reportar no sentirse satisfecho con la vida (ORa: 1.63; IC 95\%: 1.02-2.22), sentirse infeliz (ORa: 4.78; IC 95\%: 2.917.85 ), tener un mal estilo de vida (no saludable) (ORa: 2.55; IC 95\%: 1.41-4.63), autopercepción de mal estado de salud (ORa: 4.50; IC 95\%: 2.41-8.41), antecedentes de depresión (ORa: 1.99; IC 95\%: 1.33-2.97) y tener más de tres enfermedades que limitan físicamente (ORa: 3.95; IC 95\%: 2.52-6.19), en comparación con quienes se mantuvieron libres de depresión.

Para el grupo que desarrolló depresión incidente, únicamente mostraron ORa estadísticamente significativas la inseguridad alimentaria (ORa: 2.98; IC 95\%: 1.48-6.01), sentirse solo (ORa: 2.29; IC 95\%: 1.553.40) y tener tres o más enfermedades limitantes (ORa: 2.29; IC 95\%: 1.37-3.84), siendo estas variables las que se asociaron a un mayor riesgo de desarrollar depresión a 3 años de seguimiento, en comparación con quienes se mantuvieron libres de depresión en ambas mediciones.

\section{Modelo estratificado por sexo}

Cuando se separan hombres y mujeres, desarrollando un modelo para cada grupo (Tabla 3) se observan similitudes en sus ORa para depresión crónica, como lo son el caso de la ocupación, sentirse solo, sentirse infeliz, mala autopercepción de salud, antecedentes personales de depresión y presencia de enfermedades que limitan físicamente. Sin embargo, ciertas condiciones solo fueron significativas en cada uno de los sexos; así, para las mujeres, no tener escolaridad mostró una ORa de 2.34 (IC 95\%: 1.21-4.53), y de la misma manera, pertenecer al área urbana mostró una ORa de 1.83 (IC 95\%: 1.24-2.70), y la no satisfacción con la vida una ORa de 1.88 (IC 95\%: 1.31-2.70). En el caso de los hombres, reportar un estilo de vida no saludable tuvo una ORa de 3.47 (IC 95\%: 1.33-9.06). Lo mismo sucede cuando se analiza el grupo de depresión incidente por sexos: los hombres que reportaron inseguridad alimentaria tuvieron una ORa de 6.30 (IC 95\%: 2.33-17.03), entre las mujeres presentaron un mayor riesgo quienes tuvieron tres o más enfermedades limitantes (ORa: 3.32; IC 95\%: 1.66-6.62), y para ambos casos (hombres y mujeres) destaca el riesgo de desarrollar depresión incidente cuando existe el sentimiento de soledad, para los hombres con una ORa de 2.16 (IC 95\%: 1.04-4.49) y para las mujeres con una ORa un poco mayor, de 2.58 (IC 95\%: 1.64-4.07); todo esto en comparación con quienes no presentaron depresión.

\section{Perfiles de riesgo}

Finalmente, se analizaron por separado los perfiles de riesgo para pertenecer a los diferentes grupos de 
A.L. Sosa-Ortiz, et al.: Determinantes asociados a depresión

Tabla 2. Riesgos simples y ajustados para depresión crónica e incidente de la muestra total

\begin{tabular}{|c|c|c|c|c|c|}
\hline \multirow[t]{2}{*}{ Variable } & \multirow[t]{2}{*}{ Categorías } & \multicolumn{2}{|c|}{ Depresión crónica* } & \multicolumn{2}{|c|}{ Depresión incidente ${ }^{*}$} \\
\hline & & $\begin{array}{l}\text { Riesgos } \\
\text { simples }\end{array}$ & $\begin{array}{c}\text { Riesgo } \\
\text { ajustado }^{\dagger}\end{array}$ & $\begin{array}{l}\text { Riesgos } \\
\text { simples }\end{array}$ & $\begin{array}{c}\text { Riesgo } \\
\text { ajustado }^{\dagger}\end{array}$ \\
\hline Mujeres vs. hombres & & $\begin{array}{c}2.47 \\
(1.96-3.12)\end{array}$ & $\begin{array}{c}2.65 \\
(1.67-4.21)\end{array}$ & $\begin{array}{c}1.44 \\
(1.06-1.96)\end{array}$ & $\begin{array}{c}1.07 \\
(0.65-1.76)\end{array}$ \\
\hline \multirow{4}{*}{$\begin{array}{l}\text { Grupos de edad al inicio del } \\
\text { seguimiento }\end{array}$} & $65-69$ & 1.00 & 1.00 & 1.00 & 1.00 \\
\hline & $70-74$ & $\begin{array}{c}1.02 \\
(0.77-1.35)\end{array}$ & $\begin{array}{c}0.80 \\
(0.56-1.15)\end{array}$ & $\begin{array}{c}1.20 \\
(0.8-1.78)\end{array}$ & $\begin{array}{c}1.11 \\
(0.73-1.69)\end{array}$ \\
\hline & $75-79$ & $\begin{array}{c}1.19 \\
(0.87-1.63)\end{array}$ & $\begin{array}{c}0.89 \\
(0.58-1.37)\end{array}$ & $\begin{array}{c}1.30 \\
(0.84-2.03)\end{array}$ & $\begin{array}{c}1.12 \\
(0.69-1.81)\end{array}$ \\
\hline & $80+$ & $\begin{array}{c}1.20 \\
(0.88-1.66)\end{array}$ & $\begin{array}{c}0.81 \\
(0.52-1.25)\end{array}$ & $\begin{array}{c}1.13 \\
(0.72-1.79)\end{array}$ & $\begin{array}{c}0.94 \\
(0.56-1.58)\end{array}$ \\
\hline \multirow[t]{4}{*}{ Escolaridad } & Media o superior & 1.00 & 1.00 & 1.00 & 1.00 \\
\hline & $\begin{array}{l}\text { Primaria } \\
\text { completa }\end{array}$ & $\begin{array}{c}1.60 \\
(1.01-2.54)\end{array}$ & $\begin{array}{c}1.59 \\
(0.93-2.74)\end{array}$ & $\begin{array}{c}0.73 \\
(0.43-1.24)\end{array}$ & $\begin{array}{c}0.66 \\
(0.38-1.15)\end{array}$ \\
\hline & $\begin{array}{l}\text { Primaria } \\
\text { incompleta }\end{array}$ & $\begin{array}{c}2.26 \\
(1.05-3.41)\end{array}$ & $\begin{array}{c}1.98 \\
(1.18-3.32)\end{array}$ & $\begin{array}{c}1.08 \\
(0.69-1.69)\end{array}$ & $\begin{array}{c}0.82 \\
(0.51-1.34)\end{array}$ \\
\hline & Ninguna & $\begin{array}{c}3.08 \\
(2.00-4.75)\end{array}$ & $\begin{array}{c}2.31 \\
(1.33-4.02)\end{array}$ & $\begin{array}{c}1.03 \\
(0.62-1.72)\end{array}$ & $\begin{array}{c}0.75 \\
(0.42-1.31)\end{array}$ \\
\hline \multirow[t]{4}{*}{ Estado civil } & Casados & 1.00 & 1.00 & 1.00 & 1.00 \\
\hline & Solteros & $\begin{array}{c}1.14 \\
(0.68-1.92)\end{array}$ & $\begin{array}{c}0.75 \\
(0.37-1.52)\end{array}$ & $\begin{array}{c}0.97 \\
(0.48-1.93)\end{array}$ & $\begin{array}{c}0.89 \\
(0.42-1.89)\end{array}$ \\
\hline & Viudos & $\begin{array}{c}1.62 \\
(1.28-2.07)\end{array}$ & $\begin{array}{c}0.86 \\
(0.62-1.21)\end{array}$ & $\begin{array}{c}1.12 \\
(0.80-1.56)\end{array}$ & $\begin{array}{c}0.85 \\
(0.58-1.25)\end{array}$ \\
\hline & Divorciados & $\begin{array}{c}1.87 \\
(1.18-2.95)\end{array}$ & $\begin{array}{c}1.00 \\
(0.55-1.83)\end{array}$ & $\begin{array}{c}1.08 \\
(0.55-2.11)\end{array}$ & $\begin{array}{c}0.86 \\
(0.43-1.73)\end{array}$ \\
\hline \multirow[t]{4}{*}{ Ocupación } & Retirado & 1.00 & 1.00 & 1.00 & 1.00 \\
\hline & Trabaja & $\begin{array}{c}1.10 \\
(0.77-1.58)\end{array}$ & $\begin{array}{c}0.9 \\
(0.55-1.49)\end{array}$ & $\begin{array}{c}0.92 \\
(0.57-1.50)\end{array}$ & $\begin{array}{c}0.71 \\
(0.42-1.20)\end{array}$ \\
\hline & $\begin{array}{l}\text { Buscando } \\
\text { empleo }\end{array}$ & $\begin{array}{c}1.41 \\
(0.92-2.14)\end{array}$ & $\begin{array}{c}1.30 \\
(0.72-2.35)\end{array}$ & $\begin{array}{c}1.19 \\
(0.67-2.12)\end{array}$ & $\begin{array}{c}0.89 \\
(0.49-1.62)\end{array}$ \\
\hline & Hogar & $\begin{array}{c}2.47 \\
(1.82-3.37)\end{array}$ & $\begin{array}{c}1.08 \\
(0.67-1.73)\end{array}$ & $\begin{array}{c}1.80 \\
(1.18-2.73)\end{array}$ & $\begin{array}{c}1.54 \\
(0.89-2.65)\end{array}$ \\
\hline Área de residencia urbana vs. rural & & $\begin{array}{c}1.01 \\
(0.80-1.26)\end{array}$ & $\begin{array}{c}1.68 \\
(1.21-2.33)\end{array}$ & $\begin{array}{c}0.73 \\
(0.54-1.00)\end{array}$ & $\begin{array}{c}0.80 \\
(0.56-1.14)\end{array}$ \\
\hline Inseguridad alimentaria, sí vs. no & & $\begin{array}{c}3.43 \\
(2.05-5.74)\end{array}$ & $\begin{array}{c}1.96 \\
(1.03-3.73)\end{array}$ & $\begin{array}{c}3.73 \\
(2.00-6.96)\end{array}$ & $\begin{array}{c}2.98 \\
(1.48-6.01)\end{array}$ \\
\hline \multirow[t]{2}{*}{ Activos o servicios } & $\geq 4$ & 1.00 & - & 1.00 & - \\
\hline & $\leq 3$ & $\begin{array}{c}1.56 \\
(1.19-2.06)\end{array}$ & - & $\begin{array}{c}1.57 \\
(1.08-2.29)\end{array}$ & - \\
\hline \multirow[t]{3}{*}{ Contacto con familiares } & Diario & 1.00 & - & 1.00 & - \\
\hline & Semanal & $\begin{array}{c}1.15 \\
(0.85-1.55)\end{array}$ & - & $\begin{array}{c}1.41 \\
(0.96-2.07)\end{array}$ & - \\
\hline & Mensual o nunca & $\begin{array}{c}1.08 \\
(0.77-1.51)\end{array}$ & - & $\begin{array}{c}0.72 \\
(0.43-1.22)\end{array}$ & - \\
\hline
\end{tabular}


Tabla 2. Riesgos simples y ajustados para depresión crónica e incidente de la muestra total (Continued)

\begin{tabular}{|c|c|c|c|c|c|}
\hline \multirow[t]{2}{*}{ Variable } & \multirow[t]{2}{*}{ Categorías } & \multicolumn{2}{|c|}{ Depresión crónica* } & \multicolumn{2}{|c|}{ Depresión incidente* } \\
\hline & & $\begin{array}{l}\text { Riesgos } \\
\text { simples }\end{array}$ & $\begin{array}{c}\text { Riesgo } \\
\text { ajustado }^{\dagger}\end{array}$ & $\begin{array}{l}\text { Riesgos } \\
\text { simples }\end{array}$ & $\begin{array}{c}\text { Riesgo } \\
\text { ajustado }^{\dagger}\end{array}$ \\
\hline \multirow[t]{3}{*}{ Contacto con amigos } & $\begin{array}{l}\text { Semanal o más } \\
\text { frecuente }\end{array}$ & 1.00 & 1.00 & 1.00 & 1.00 \\
\hline & $\begin{array}{l}\text { Al menos una } \\
\text { vez al mes }\end{array}$ & $\begin{array}{c}1.09 \\
(0.70-1.71)\end{array}$ & $\begin{array}{c}0.96 \\
(0.54-1.69)\end{array}$ & $\begin{array}{c}1.09 \\
(0.61-1.96)\end{array}$ & $\begin{array}{c}1.20 \\
(0.66-2.17)\end{array}$ \\
\hline & Nunca & $\begin{array}{c}1.87 \\
(1.49-2.36)\end{array}$ & $\begin{array}{c}1.40 \\
(1.04-1.90)\end{array}$ & $\begin{array}{c}1.11 \\
(0.81-1.52)\end{array}$ & $\begin{array}{c}1.02 \\
(0.73-1.42)\end{array}$ \\
\hline \multirow[t]{3}{*}{ Contacto con vecinos } & $\begin{array}{l}\text { Semanal o más } \\
\text { frecuente }\end{array}$ & 1.00 & - & 1.00 & - \\
\hline & $\begin{array}{l}\text { Al menos una } \\
\text { vez al mes }\end{array}$ & $\begin{array}{c}1.18 \\
(0.86-1.64)\end{array}$ & - & $\begin{array}{c}1.05 \\
(0.67-1.63)\end{array}$ & - \\
\hline & Nunca & $\begin{array}{c}1.75 \\
(1.33-2.30)\end{array}$ & - & $\begin{array}{c}1.18 \\
(0.79-1.75)\end{array}$ & - \\
\hline $\begin{array}{l}\text { No asiste a reuniones religiosas vs. } \\
\text { asiste regular u ocasionalmente }\end{array}$ & & $\begin{array}{c}1.30 \\
(0.87-1.95)\end{array}$ & - & $\begin{array}{c}0.98 \\
(0.54-1.75)\end{array}$ & - \\
\hline $\begin{array}{l}\text { No asiste a reuniones sociales o } \\
\text { comunitarias vs. asiste regular u } \\
\text { ocasionalmente }\end{array}$ & & $\begin{array}{c}1.11 \\
(0.85-1.44)\end{array}$ & - & $\begin{array}{c}0.95 \\
(0.67-1.35)\end{array}$ & - \\
\hline Vive solo vs. vive acompañado & & $\begin{array}{c}1.57 \\
(1.08-2.27)\end{array}$ & - & $\begin{array}{c}1.40 \\
(0.84-2.32)\end{array}$ & - \\
\hline Se siente solo vs. no se siente solo & & $\begin{array}{c}8.13 \\
(6.21-10.63)\end{array}$ & $\begin{array}{c}4.63 \\
(3.34-6.44)\end{array}$ & $\begin{array}{c}2.65 \\
(1.85-3.79)\end{array}$ & $\begin{array}{c}2.29 \\
(1.55-3.40)\end{array}$ \\
\hline $\begin{array}{l}\text { Insatisfacción con la vida vs. } \\
\text { satisfacción }\end{array}$ & & $\begin{array}{c}2.72 \\
(2.16-3.43)\end{array}$ & $\begin{array}{c}1.63 \\
(1.02-2.22)\end{array}$ & $\begin{array}{c}1.27 \\
(0.94-1.73)\end{array}$ & $\begin{array}{c}1.09 \\
(0.78-1.52)\end{array}$ \\
\hline \multirow[t]{2}{*}{ Felicidad } & Feliz, muy feliz & 1.00 & 1.00 & 1.00 & 1.00 \\
\hline & Poco o nada feliz & $\begin{array}{c}12.44 \\
(8.16-18.97)\end{array}$ & $\begin{array}{c}4.78 \\
(2.91-7.85)\end{array}$ & $\begin{array}{c}1.57 \\
(0.82-3.01)\end{array}$ & $\begin{array}{c}1.10 \\
(0.55-2.18)\end{array}$ \\
\hline \multirow[t]{3}{*}{ Estilo de vida } & $\begin{array}{l}\text { Adecuado o } \\
\text { ideal }\end{array}$ & 1.0 & 1.00 & 1.00 & 1.00 \\
\hline & Mejorable & $\begin{array}{c}2.17 \\
(1.62-2.92)\end{array}$ & $\begin{array}{c}1.70 \\
(1.16-2.50)\end{array}$ & $\begin{array}{c}1.20 \\
(0.82-1.74)\end{array}$ & $\begin{array}{c}1.08 \\
(0.73-1.62)\end{array}$ \\
\hline & Inadecuado & $\begin{array}{c}2.96 \\
(1.93-4.56)\end{array}$ & $\begin{array}{c}2.55 \\
(1.41-4.63)\end{array}$ & $\begin{array}{c}1.30 \\
(0.72-2.35)\end{array}$ & $\begin{array}{c}1.44 \\
(0.76-2.73)\end{array}$ \\
\hline \multirow[t]{3}{*}{ Autopercepción de salud } & Buena & 1.00 & 1.00 & 1.00 & 1.00 \\
\hline & Regular & $\begin{array}{c}3.23 \\
(2.54-4.11)\end{array}$ & $\begin{array}{c}2.11 \\
(1.57-2.85)\end{array}$ & $\begin{array}{c}0.98 \\
(0.71-1.37)\end{array}$ & $\begin{array}{c}0.83 \\
(0.59-1.18)\end{array}$ \\
\hline & Mala & $\begin{array}{c}9.80 \\
(6.01-15.99)\end{array}$ & $\begin{array}{c}4.50 \\
(2.41-8.41)\end{array}$ & $\begin{array}{c}1.87 \\
(0.93-3.76)\end{array}$ & $\begin{array}{c}1.55 \\
(0.73-3.30)\end{array}$ \\
\hline $\begin{array}{l}\text { Alteraciones cognitivas en el } \\
\text { desarrollo }\end{array}$ & & $\begin{array}{c}3.99 \\
(1.97-8.08)\end{array}$ & - & $\begin{array}{c}1.74 \\
(0.63-4.85)\end{array}$ & - \\
\hline Deterioro cognitivo & & $\begin{array}{c}2.59 \\
(1.81-3.71)\end{array}$ & - & $\begin{array}{c}1.19 \\
(0.69-2.06)\end{array}$ & - \\
\hline Antecedentes de depresión & & $\begin{array}{c}2.84 \\
(2.10-3.86)\end{array}$ & $\begin{array}{c}1.99 \\
(1.33-2.97)\end{array}$ & $\begin{array}{c}0.92 \\
(0.56-1.52)\end{array}$ & $\begin{array}{c}0.79 \\
(0.46-1.34)\end{array}$ \\
\hline
\end{tabular}


A.L. Sosa-Ortiz, et al.: Determinantes asociados a depresión

Tabla 2. Riesgos simples y ajustados para depresión crónica e incidente de la muestra total (Continued)

\begin{tabular}{|c|c|c|c|c|c|}
\hline \multirow[t]{2}{*}{ Variable } & \multirow[t]{2}{*}{ Categorías } & \multicolumn{2}{|c|}{ Depresión crónica* } & \multicolumn{2}{|c|}{ Depresión incidente* } \\
\hline & & $\begin{array}{l}\text { Riesgos } \\
\text { simples }\end{array}$ & $\begin{array}{c}\text { Riesgo } \\
\text { ajustado }^{\dagger}\end{array}$ & $\begin{array}{l}\text { Riesgos } \\
\text { simples }\end{array}$ & $\begin{array}{c}\text { Riesgo } \\
\text { ajustado }^{\dagger}\end{array}$ \\
\hline \multirow[t]{3}{*}{ Enfermedades que limitan físicamente } & Ninguna & 1.00 & 1.00 & 1.00 & 1.00 \\
\hline & Una o dos & $\begin{array}{c}2.31 \\
(1.80-2.98)\end{array}$ & $\begin{array}{c}1.86 \\
(1.37-2.54)\end{array}$ & $\begin{array}{c}1.35 \\
(0.97-1.88)\end{array}$ & $\begin{array}{c}1.34 \\
(0.94-1.89)\end{array}$ \\
\hline & Tres o más & $\begin{array}{c}7.93 \\
(5.5-11.44)\end{array}$ & $\begin{array}{c}3.95 \\
(2.52-6.19)\end{array}$ & $\begin{array}{c}2.49 \\
(1.52-4.08)\end{array}$ & $\begin{array}{c}2.29 \\
(1.37-3.84)\end{array}$ \\
\hline
\end{tabular}

*El modelo multinomial tiene como referencia el grupo de sujetos sin depresión.

tLos modelos ajustados incluyeron las variables sexo, grupos de edad al inicio del seguimiento, escolaridad, estado civil, ocupación, área de residencia, inseguridad alimentaria,

contacto con amigos, se siente solo, satisfacción con la vida, felicidad, estilo de vida (alcoholismo, tabaquismo y actividad física), autopercepción de salud, antecedentes de depresión

y enfermedades que limitan físicamente.

Tabla 3. Riesgos ajustados para depresión crónica e incidente por sexo*

\begin{tabular}{|c|c|c|c|c|c|}
\hline \multirow[t]{3}{*}{ Variable } & \multirow[t]{3}{*}{ Categorías } & \multicolumn{2}{|c|}{ Hombres } & \multicolumn{2}{|c|}{ Mujeres } \\
\hline & & $\begin{array}{c}\text { Depresión } \\
\text { crónica }^{\dagger}\end{array}$ & $\begin{array}{l}\text { Depresión } \\
\text { incidente }^{\dagger}\end{array}$ & $\begin{array}{c}\text { Depresión } \\
\text { crónica }^{\dagger}\end{array}$ & $\begin{array}{l}\text { Depresión } \\
\text { incidente }^{\dagger}\end{array}$ \\
\hline & & $\begin{array}{c}\text { Riesgo } \\
\text { ajustado }\end{array}$ & Riesgo ajustado & $\begin{array}{c}\text { Riesgo } \\
\text { ajustado }^{\ddagger}\end{array}$ & $\begin{array}{c}\text { Riesgo } \\
\text { ajustado }\end{array}$ \\
\hline \multirow{4}{*}{$\begin{array}{l}\text { Grupos de edad al inicio del } \\
\text { seguimiento }\end{array}$} & $65-69$ & 1.00 & 1.00 & 1.00 & 1.00 \\
\hline & $70-74$ & $\begin{array}{c}0.72 \\
(0.34-1.54)\end{array}$ & $\begin{array}{c}1.33 \\
(0.62-2.85)\end{array}$ & $\begin{array}{c}0.93 \\
(0.60-1.45)\end{array}$ & $\begin{array}{c}1.09 \\
(0.65-1.83)\end{array}$ \\
\hline & $75-79$ & $\begin{array}{c}1.42 \\
(0.62-3.23)\end{array}$ & $\begin{array}{c}1.74 \\
(0.75-4.01)\end{array}$ & $\begin{array}{c}0.75 \\
(0.44-1.26)\end{array}$ & $\begin{array}{c}0.88 \\
(0.48-1.61)\end{array}$ \\
\hline & $80+$ & $\begin{array}{c}0.72 \\
(0.30-1.78)\end{array}$ & $\begin{array}{c}1.37 \\
(0.58-3.23)\end{array}$ & $\begin{array}{c}0.91 \\
(0.54-1.54)\end{array}$ & $\begin{array}{c}0.79 \\
(0.41-1.52)\end{array}$ \\
\hline \multirow[t]{4}{*}{ Escolaridad } & Media o superior & 1.00 & 1.00 & 1.00 & 1.00 \\
\hline & $\begin{array}{l}\text { Primaria } \\
\text { completa }\end{array}$ & $\begin{array}{c}1.58 \\
(0.53-4.71)\end{array}$ & $\begin{array}{c}0.75 \\
(0.31-1.79)\end{array}$ & $\begin{array}{c}1.39 \\
(0.71-2.72)\end{array}$ & $\begin{array}{c}0.57 \\
(0.26-1.22)\end{array}$ \\
\hline & $\begin{array}{l}\text { Primaria } \\
\text { incompleta }\end{array}$ & $\begin{array}{c}2.37 \\
(0.87-6.42)\end{array}$ & $\begin{array}{c}0.78 \\
(0.37-1.64)\end{array}$ & $\begin{array}{c}1.86 \\
(1.01-3.45)\end{array}$ & $\begin{array}{c}0.84 \\
(0.44-1.59)\end{array}$ \\
\hline & Ninguna & $\begin{array}{c}2.65 \\
(0.90-7.81)\end{array}$ & $\begin{array}{c}0.61 \\
(0.24-1.58)\end{array}$ & $\begin{array}{c}2.34 \\
(1.21-4.53)\end{array}$ & $\begin{array}{c}0.82 \\
(0.40-1.68)\end{array}$ \\
\hline \multirow[t]{4}{*}{ Estado civil } & Casados & 1.00 & 1.00 & 1.00 & 1.00 \\
\hline & Solteros & $\begin{array}{c}0.92 \\
(0.35-2.41)\end{array}$ & $\begin{array}{c}1.03 \\
(0.28-3.74)\end{array}$ & $\begin{array}{c}0.79 \\
(0.35-1.80)\end{array}$ & $\begin{array}{c}1.03 \\
(0.43-2.48)\end{array}$ \\
\hline & Viudos & $\begin{array}{c}0.61 \\
(0.12-3.04)\end{array}$ & $\begin{array}{c}0.64 \\
(0.09-4.43)\end{array}$ & $\begin{array}{c}0.82 \\
(0.56-1.20)\end{array}$ & $\begin{array}{c}0.89 \\
(0.56-1.41)\end{array}$ \\
\hline & Divorciados & $\begin{array}{c}0.89 \\
(0.03-2.64)\end{array}$ & $\begin{array}{c}0.84 \\
(0.20-3.43)\end{array}$ & $\begin{array}{c}1.09 \\
(0.55-2.18)\end{array}$ & $\begin{array}{c}0.94 \\
(0.42-2.11)\end{array}$ \\
\hline \multirow[t]{3}{*}{ Ocupación } & Retirado & $\begin{array}{c}2.58 \\
(1.33-5.00)\end{array}$ & $\begin{array}{c}1.51 \\
(0.08-2.85)\end{array}$ & 1.00 & 1.00 \\
\hline & Trabaja & 1.00 & 1.00 & $\begin{array}{c}2.21 \\
(1.05-4.65)\end{array}$ & $\begin{array}{c}1.05 \\
(0.40-2.70)\end{array}$ \\
\hline & $\begin{array}{l}\text { Buscando } \\
\text { empleo }\end{array}$ & $\begin{array}{c}1.51 \\
(0.77-2.98)\end{array}$ & $\begin{array}{c}1.23 \\
(0.63-2.39)\end{array}$ & $\begin{array}{c}4.96 \\
(1.28-19.20)\end{array}$ & $\begin{array}{c}1.50 \\
(0.24-9.38)\end{array}$ \\
\hline
\end{tabular}


Tabla 3. Riesgos ajustados para depresión crónica e incidente por sexo* (Continued)

\begin{tabular}{|c|c|c|c|c|c|}
\hline \multirow[t]{3}{*}{ Variable } & \multirow[t]{3}{*}{ Categorías } & \multicolumn{2}{|c|}{ Hombres } & \multicolumn{2}{|c|}{ Mujeres } \\
\hline & & $\begin{array}{l}\text { Depresión } \\
\text { crónica }^{\dagger}\end{array}$ & $\begin{array}{l}\text { Depresión } \\
\text { incidente }^{\dagger}\end{array}$ & $\begin{array}{l}\text { Depresión } \\
\text { crónica }^{\dagger}\end{array}$ & $\begin{array}{l}\text { Depresión } \\
\text { incidente }^{\dagger}\end{array}$ \\
\hline & & $\begin{array}{l}\text { Riesgo } \\
\text { ajustado¥ }\end{array}$ & Riesgo ajustado ${ }^{\ddagger}$ & $\begin{array}{l}\text { Riesgo } \\
\text { ajustado }\end{array}$ & $\begin{array}{c}\text { Riesgo } \\
\text { ajustado }\end{array}$ \\
\hline & Hogar & $\begin{array}{c}3.01 \\
(0.48-19.04)\end{array}$ & $\begin{array}{c}1.85 \\
(0.18-19.48)\end{array}$ & $\begin{array}{c}1.99 \\
(1.07-3.72)\end{array}$ & $\begin{array}{c}1.94 \\
(0.92-4.09)\end{array}$ \\
\hline $\begin{array}{l}\text { Área de residencia urbana } \\
\text { vs. rural }\end{array}$ & & - & - & $\begin{array}{c}1.83 \\
(1.24-2.70)\end{array}$ & $\begin{array}{c}0.78 \\
(0.49-1.22)\end{array}$ \\
\hline $\begin{array}{l}\text { Inseguridad alimentaria (sí } \\
\text { vs. no) }\end{array}$ & & $\begin{array}{c}2.29 \\
(0.81-6.47)\end{array}$ & $\begin{array}{c}6.30 \\
(2.33-17.03)\end{array}$ & - & - \\
\hline $\begin{array}{l}\text { Se siente solo vs. no se siente } \\
\text { solo }\end{array}$ & & $\begin{array}{c}5.85 \\
(3.12-10.97)\end{array}$ & $\begin{array}{c}2.16 \\
(1.04-4.49)\end{array}$ & $\begin{array}{c}5.00 \\
(3.41-7.34)\end{array}$ & $\begin{array}{c}2.58 \\
(1.64-4.07)\end{array}$ \\
\hline $\begin{array}{l}\text { Insatisfacción con la vida vs. } \\
\text { satisfacción }\end{array}$ & & - & - & $\begin{array}{c}1.88 \\
(1.31-2.70)\end{array}$ & $\begin{array}{c}1.09 \\
(0.72-1.66)\end{array}$ \\
\hline \multirow[t]{2}{*}{ Felicidad } & Feliz, muy feliz & 1.00 & 1.00 & 1.00 & 1.00 \\
\hline & $\begin{array}{l}\text { Poco o nada } \\
\text { feliz }\end{array}$ & $\begin{array}{c}3.60 \\
(1.61-8.04)\end{array}$ & $\begin{array}{c}0.89 \\
(0.28-2.77)\end{array}$ & $\begin{array}{c}6.21 \\
(3.04-12.71)\end{array}$ & $\begin{array}{c}1.26 \\
(0.49-3.21)\end{array}$ \\
\hline \multirow[t]{3}{*}{ Estilo de vida } & $\begin{array}{l}\text { Adecuado o } \\
\text { ideal }\end{array}$ & 1.00 & 1.00 & - & - \\
\hline & Mejorable & $\begin{array}{c}3.10 \\
(1.33-7.25)\end{array}$ & $\begin{array}{c}1.59 \\
(0.75-3.36)\end{array}$ & - & - \\
\hline & Inadecuado & $\begin{array}{c}3.47 \\
(1.33-9.06)\end{array}$ & $\begin{array}{c}1.58 \\
(0.59-4.22)\end{array}$ & - & - \\
\hline \multirow[t]{3}{*}{ Autopercepción de salud } & Buena & 1.00 & 1.00 & 1.00 & 1.00 \\
\hline & Regular & $\begin{array}{c}2.17 \\
(1.24-3.78)\end{array}$ & $\begin{array}{c}0.82 \\
(0.46-1.46)\end{array}$ & $\begin{array}{c}2.35 \\
(1.63-3.39)\end{array}$ & $\begin{array}{c}0.90 \\
(0.58-1.40)\end{array}$ \\
\hline & Mala & $\begin{array}{c}3.39 \\
(1.20-9.57)\end{array}$ & $\begin{array}{c}1.78 \\
(0.53-5.97)\end{array}$ & $\begin{array}{c}6.20 \\
(2.86-13.45)\end{array}$ & $\begin{array}{c}1.79 \\
(0.68-4.70)\end{array}$ \\
\hline Antecedentes de depresión & & $\begin{array}{c}2.36 \\
(1.16-4.77)\end{array}$ & $\begin{array}{c}0.29 \\
(0.07-1.20)\end{array}$ & $\begin{array}{c}2.02 \\
(1.23-3.32)\end{array}$ & $\begin{array}{c}1.07 \\
(0.59-1.96)\end{array}$ \\
\hline \multirow{3}{*}{$\begin{array}{l}\text { Enfermedades que limitan } \\
\text { físicamente }\end{array}$} & Ninguna & 1.00 & 1.00 & 1.00 & 1.00 \\
\hline & Una o dos & $\begin{array}{c}3.55 \\
(1.90-6.63)\end{array}$ & $\begin{array}{c}1.54 \\
(0.88-2.72)\end{array}$ & $\begin{array}{c}1.52 \\
(1.06-2.19)\end{array}$ & $\begin{array}{c}1.20 \\
(0.78-1.86)\end{array}$ \\
\hline & Tres o más & $\begin{array}{c}4.38 \\
(2.01-9.52)\end{array}$ & $\begin{array}{c}1.85 \\
(0.79-4.34)\end{array}$ & $\begin{array}{c}4.55 \\
(2.42-8.55)\end{array}$ & $\begin{array}{c}3.32 \\
(1.66-6.62)\end{array}$ \\
\hline
\end{tabular}

*Se muestran los modelos finales que mostraron mejor ajuste.

tEl modelo multinomial tiene como referencia el grupo de sujetos sin depresión.

‡Los modelos ajustados incluyeron las variables grupo de edad al inicio del seguimiento, escolaridad, estado civil, ocupación, área de residencia, inseguridad alimentaria, se siente solo, satisfacción con la vida, felicidad, estilo de vida (alcoholismo, tabaquismo y actividad física), autopercepción de salud, antecedentes de depresión y enfermedades que limitan físicamente.

depresión (crónica, incidente y sin depresión), para hombres y mujeres (Figs. 2 y 3 ). Primero se ilustra el riesgo individual de cada una de las condiciones evaluadas, y después adicionando las condiciones más prevalentes. El total de hombres o mujeres hace referencia a la probabilidad global por sexo de pertenecer a cada uno de los grupos de depresión.
Entre las mujeres se observó una probabilidad del $53 \%$ de pertenecer al grupo de depresión crónica, del $16 \%$ para el grupo de depresión incidente y del 31\% para mantenerse libre de dicha condición (Fig. 3); mientras que los hombres presentaron un riesgo del $23 \%$ para depresión crónica, del 17\% para depresión incidente y del $60 \%$ para permanecer libres de 


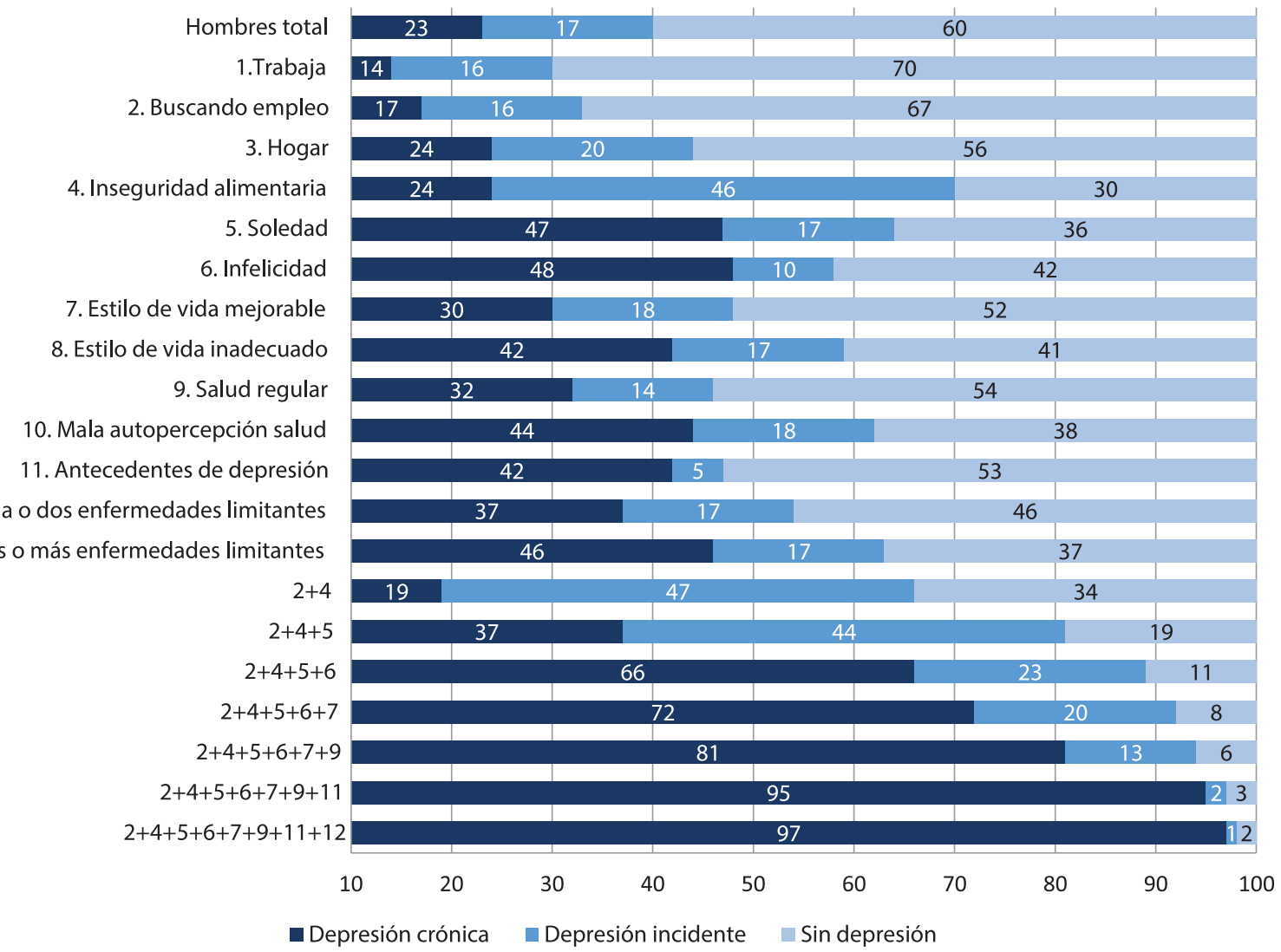

Figura 2. Perfil de riesgos para depresión en hombres.

depresión (Fig. 2). Por otra parte, las probabilidades acumuladas se comportan de forma heterogénea entre los grupos de depresión y entre los sexos; por ejemplo, en los hombres, el riesgo de pertenecer al grupo de depresión crónica aumentó al $66 \%$ cuando se consideraron la ocupación, la inseguridad alimentaria, la soledad y la infelicidad; y el riesgo para depresión incidente aumentó del 17 al $47 \%$ cuando se dan solo dos condiciones: buscar trabajo y situación de inseguridad alimentaria. En el caso de las mujeres, los mayores riesgos se observaron para la depresión crónica, destacando la situación de búsqueda de empleo, con un riesgo individual del $82 \%$, el cual es superado únicamente por la autopercepción de mal estado de salud $(83 \%)$.

\section{Discusión}

Este trabajo demuestra que la depresión en los AM mexicanos, al igual que en otros países, es un fenómeno cuya presentación o persistencia (crónica o incidente) está asociada a múltiples factores sociodemográficos, psicosociales y de salud.
Del total de la muestra estudiada, el $57 \%$ presentó síntomas depresivos, ya sea como depresión crónica o incidente. En una revisión sistemática de estudios con modelamiento de trayectorias de depresión en población general, la cual consistió en una revisión de 24 estudios de cohorte, encontraron una prevalencia del $10 \%$ de síntomas depresivos persistentes $^{22}$, en contraste con el $42 \%$ de depresión crónica reportada en este estudio, cifra que es también superior a las de otros estudios longitudinales de depresión crónica en AM, como el de Diegelmann, et al. ${ }^{23}$, quienes reportaron, en una muestra de 2219 AM evaluados en cinco ocasiones entre 2002 y 2011, que un $22 \%$ se mantuvo con depresión persistente y un $32 \%$ no presentaron depresión, mientras que un $46 \%$ incrementaron la sintomatología con el paso del tiempo.

La depresión crónica mostró estar asociada con un mayor número de condiciones, en comparación con la depresión incidente, estando esta última más relacionada con situaciones de pobreza, soledad y pérdida de salud, las cuales acompañan con frecuencia al proceso de envejecer. 


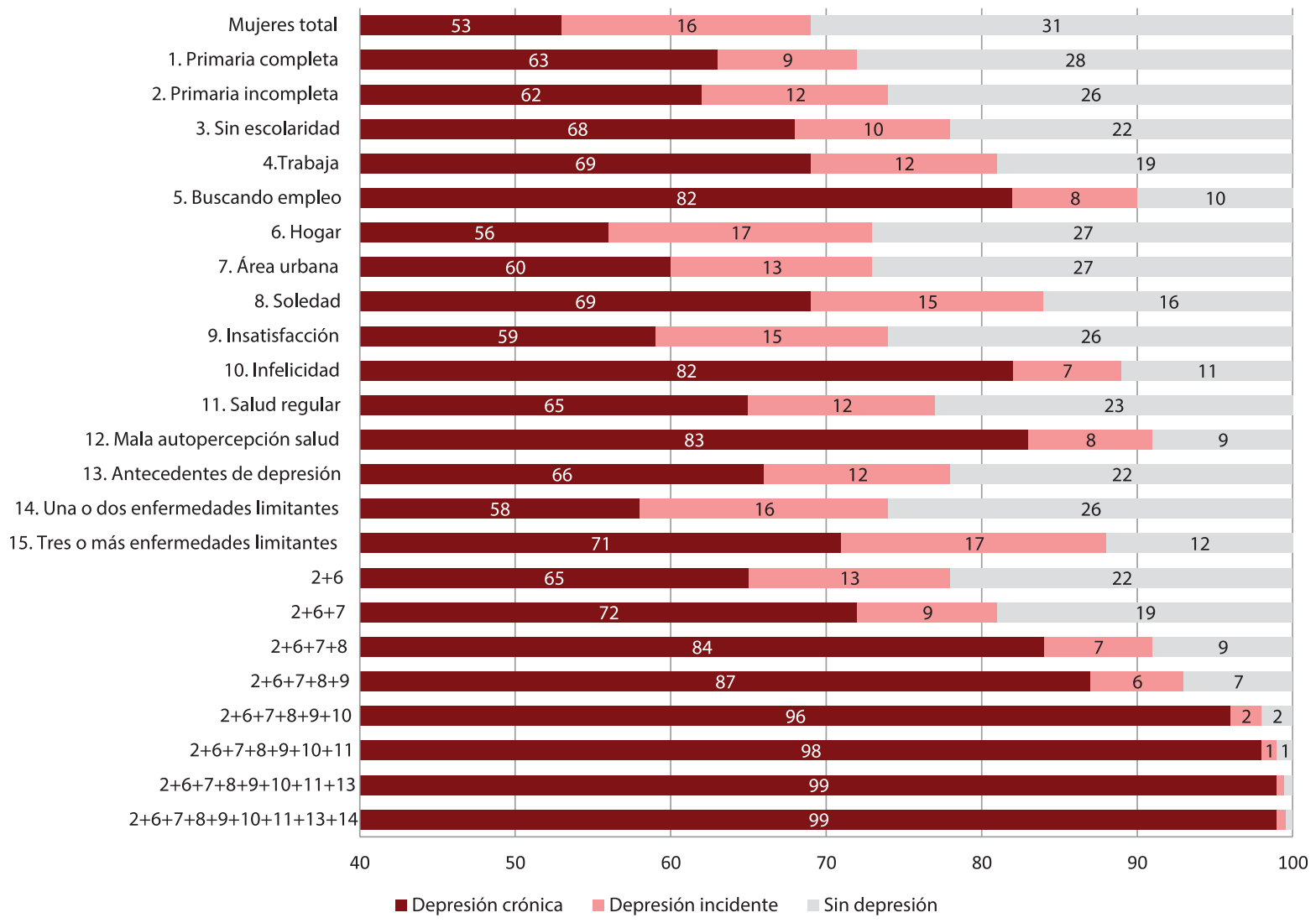

Figura 3. Perfil de riesgos para depresión en mujeres.

Nuestros resultados muestran a la soledad como un factor de riesgo consistente, en las condiciones de depresión tanto crónica como incidente, así como para ambos sexos. La soledad en el AM también ha sido relacionada con otros desenlaces no deseados. Por ejemplo, en un estudio de seguimiento a 2 años de 285 AM entre 60 y 90 años, con diagnóstico de depresión mayor, distimia o síntomas depresivos, se reportó que la soledad se asoció de forma independiente con síntomas depresivos graves en el seguimiento, y una forma grave de esta se asoció con un mal pronóstico para su remisión, en comparación con quienes no la presentaban (ORa: 0.25; IC 95\%: $0.08-0.80)^{24}$.

Por otro lado, se ha reportado que el incrementar el número de grupos sociales a los que puede pertenecer el AM se reduce el riesgo de recaída de depresión en un $24 \%$, y si se está en contacto con tres grupos o más, este riesgo disminuye un $63 \%$, lo que sugiere que la pertenencia a grupos sociales puede ser tanto un factor protector como una intervención efectiva contra la depresión. Así lo demostró un estudio con 4087 AM y seguimiento de 4 años realizado por Cruwys, et al. ${ }^{25}$. Sin embargo, debe estudiarse más ampliamente la contribución de los amigos, pues en este estudio, aunque parecía ser un aspecto importante en el modelo general, su efecto desapareció al estratificar por sexo.

Por otro lado, al comparar las diferentes condiciones de depresión por sexo se observó una mayor prevalencia en las mujeres, lo cual ha sido repetidamente reportado $22,26,27$. Además, se encontró que la baja escolaridad es un factor que tiene un mayor impacto en ellas y que se asocia a depresión crónica. Con respecto a la ocupación, mientras que trabajar se presenta como una condición de protección para el hombre, para la mujer el trabajar, el dedicarse al hogar, y estar en búsqueda de empleo, se comportaron como condiciones de riesgo, probablemente por estar asociadas a condiciones de aparición tardía del proveedor, tales como pérdida de ingresos, carencia de pensión por jubilación o muerte. El impacto negativo del desempleo en hombres ya ha sido documentado ${ }^{28,29}$, y se han dado distintas explicaciones, entre las que se ha considerado que pudiera tener relación con los roles sociales, el ingreso y factores psicológicos tales como 
la identidad, la autoestima, la autopercepción, las expectativas y las preferencias ${ }^{29}$.

La inseguridad alimentaria mostró ser un factor de riesgo para la depresión incidente en los hombres, lo que probablemente pueda estar asociado a la disminución de ingresos, por lo que se debe considerar el impacto de una situación económica deteriorada, aunada a otras pérdidas propias del envejecer, como la disminución del estatus. En los AM, cuando esta condición incrementa, amenaza a su salud y funcionalidad ${ }^{30}$.

Las diferencias encontradas por sexo dan cuenta del contexto social en el que se desenvuelven los AM, y de cómo el sexo impacta diferencialmente. En una cohorte de 4049 AM de Taiwán, seguidos durante 18 años, se reportó que las diferencias de síntomas depresivos por sexo se explicaban por la posición social y el empleo, y que tener una posición social baja puede hacer aún más vulnerables a las mujeres ${ }^{26}$. Trasladando este hallazgo a nuestros resultados, es de esperar que las desventajas sociales en la mujer se asocien a una baja escolaridad y a la residencia rural, ambos factores de riesgo para depresión crónica.

Con respecto al estado de salud, es importante reconocer que en los AM con alguna enfermedad los síntomas depresivos pueden ser pasados por alto, ya que muchas veces se relacionan con la enfermedad médica concurrente, problemas de funcionalidad 0 , peor aún, pueden ser vistos como propios de la edad, pues muchos de los síntomas de la depresión, tales como disminución de la energía, fatiga, pérdida de apetito y alteraciones del sueño, son síntomas que con frecuencia se asocian a enfermedades somáticas. Además, se debe considerar que el ánimo deprimido comórbido puede retrasar o impedir que se busque o se reciba la atención médica necesaria para dicha condición. Nuestro estudio mostró que la comorbilidad que limita físicamente tuvo un mayor impacto en las mujeres, siendo esta condición una de las dos variables que se asociaron a la depresión incidente, no así en los hombres. También es importante recordar que, en general, las mujeres envejecen en peores condiciones de salud que los hombres ${ }^{11,26}$.

Con respecto a los perfiles de riesgo, se observó que las condiciones asociadas a la pertenencia al grupo de depresión crónica o incidente fue diferente entre hombres y mujeres, destacando el caso del papel de la inseguridad alimentaria para el desarrollo de depresión incidente en hombres, incluso equiparándose al de la suma o combinación de dos o más condiciones.

El tratamiento de la depresión impacta positivamente la salud de los AM. Se ha reportado que su tratamiento mejora el funcionamiento físico de los AM, incluso en presencia de enfermedades crónicas ${ }^{31,32}$, llegando a revertir incluso condiciones de discapacidad $^{33}$. No obstante, es una realidad que, en los países de bajo y mediano ingreso, la posibilidad de recibir tratamiento oportuno y adecuado es limitada ${ }^{32}$. En México, en una encuesta realizada a 2322 AM derechohabientes del Instituto Mexicano del Seguro Social, se encontró que solo el $7.6 \%$ de los sujetos con síntomas depresivos contaba con atención adecuada, lo cual se traduce en que más del $90 \%$ no la recibe, ya sea porque no la solicitan o porque no se les otorga $^{34}$.

Por todo lo hasta aquí señalado, debe considerarse que es necesario identificar las diversas condiciones asociadas a cada tipo particular de depresión e identificar grupos en riesgo para planear intervenciones, para contribuir a abatir la depresión en el AM y sus consecuencias. De entre ellas podemos mencionar la implementación de actividades para mejorar el contacto social, o la práctica de actividades físicas en grupo, las cuales han demostrado tener un efecto positivo en los AM con depresión ${ }^{35}$.

Entre las limitaciones del presente trabajo debe señalarse que, si bien la población estudiada carece de representatividad nacional, la distribución de las características generales de esta población es similar a la reportada en encuestas nacionales, como la ENSANUT ${ }^{36}$ y la ENASEM ${ }^{37,38}$. Otra consideración es que mediciones como la soledad, la insatisfacción o la infelicidad pueden están relacionadas con el constructo de la depresión.

Entre las fortalezas de este trabajo es importante recordar que los datos provienen de un estudio diseñado específicamente para evaluar un número importante de condiciones de salud física y mental del AM, por medio de evaluaciones e instrumentos estandarizados, que se aplicaron tanto al participante como a un informante.

En resumen, la trayectoria de la depresión es heterogénea, lo cual puede indicar que hay diferencias en su etiología y naturaleza. Atender a grupos de AM en riesgo de presentar depresión es una tarea pendiente. Las trayectorias de la depresión crónica pueden predecir discapacidad y mortalidad, incluso en AM aparentemente sanos ${ }^{39}$. 
En conclusión, se debe hacer énfasis en la identificación y el monitoreo de los síntomas depresivos en el AM, así como en el conocimiento y la detección de los factores de riesgo específicos para hombres y mujeres ${ }^{27}$, para poder incidir sobre ellos oportunamente y contribuir a incrementar la calidad de vida y los años vividos con salud de los AM.

\section{Bibliografía}

1. Sosa Ortiz AL, Acosta Castillo GI. La salud mental del adulto mayor. En: Medina Mora ME, Sarti Gutiérrez EJ, Real Quintanar T, editores. La depresión y otros trastornos psiquiátricos. México: Intersistemas Editores; 2015. p. 101-12.

2. Vega García E, González Picos J, Llibre Rodríguez J, et al. Trastornos mentales en los adultos mayores. En: Epidemiología de los trastornos mentales en América Latina y el Caribe. Washington, D.C.: Publicación Cientifica y Técnica; 2009. p. 243-55

3. Sousa RM, Ferri CP, Acosta D, et al. Contribution of chronic diseases to disability in elderly people in countries with low and middle incomes: a 10/66 Dementia Research Group population-based survey. Lancet. 2009;374:1821-30.

4. Prince M, Patel V, Saxena S, et al. No health without mental health. Lancet. 2007;370:859-77.

5. Glover J, Srinivasan S. Assessment of the person with late-life depression. Psychiatr Clin N Am. 2013;36:545-60.

6. Licht-Strunk E, van der Windt DA, van Marwijk HW, et al. The prognosis of depression in older patients in general practice and the community. A systematic review. Fam Pract. 2007;24:168-80.

7. Andreescu C, Chang CC, Mulsant BH, et al. Twelve-year depressive symptom trajectories and their predictors in a community sample of older adults. Int Psychogeriat. 2008;20:221-36.

8. Kuchibhatla MN, Fillenbaum GG, Hybels CF, et al. Trajectory classes of depressive symptoms in a community sample of older adults. Acta Psychiatr Scand. 2012;125:492-501.

9. Liang J, Xu X, Quinones AR, et al. Multiple trajectories of depressive symptoms in middle and late life: racial/ethnic variations. Psychol Aging. 2011;26:761-77.

10. Hsu HC. Group-based trajectories of depressive symptoms and the predictors in the older population. Int J Geriatr Psychiatr. 2012;27:854-62.

11. Burns RA, Luszcz MA, Kiely KM, et al. Gender differences in the trajectories of late-life depressive symptomology and probable depression in the years prior to death. Int Psychogeriatr. 2013;25:1765-73.

12. Social determinants of mental health. Geneva, Switzerland: World Health Organization; 2014

13. Prince M, Ferri CP, Acosta D, et al. The protocols for the 10/66 dementia research group population-based research programme. BMC Public Health. 2007:7:165

14. Prina AM, Acosta D, Acostas I, et al. Cohort profile: the 10/66 study. Int J Epidemiol. 2017;46:406-406i.

15. Copeland JR, Dewey ME, Henderson AS, et al. The Geriatric Mental State (GMS) used in the community: replication studies of the computerized diagnosis AGECAT. Psychol Med. 1988;18:219-23.

16. Guerra M, Ferri CP, Sosa AL, et al. Late-life depression in Peru, Mexico and Venezuela: the 10/66 population-based study. Br J Psychiatry. 2009;195:510-5.

17. Ferri CP, West R, Moriyama TS, et al. Tobacco use and dementia: evidence from the 1066 dementia population-based surveys in Latin America, China and India. Int J Geriatr Psychiatry. 2011;26:1177-85.

18. Sosa AL, Albanese E, Stephan BC, et al. Prevalence, distribution, and impact of mild cognitive impairment in Latin America, China, and India: a 10/66 population-based study. PLoS Med. 2012;9:e1001170.
19. Prince $M$, Acosta $D$, Chiu $H$, et al. Dementia diagnosis in developing countries: a cross-cultural validation study. Lancet. 2003;361:909-17.

20. Prince MJ, de Rodríguez JL, Noriega L, et al. The 10/66 Dementia Research Group's fully operationalised DSM-IV dementia computerized diagnostic algorithm, compared with the 10/66 dementia algorithm and a clinician diagnosis: a population validation study. BMC Public Health. 2008;8:219

21. Frome EL, Morris MD. Evaluating goodness of fit of poisson regression models in cohort studies. The American Statistician. 1989;43:144-7.

22. Musliner KL, Munk-Olsen T, Eaton WW, et al. Heterogeneity in long-term trajectories of depressive symptoms: patterns, predictors and outcomes. Jffect Dis. 2016;192:199-211.

23. Diegelmann M, Schilling OK, Wahl HW. Feeling blue at the end of life: trajectories of depressive symptoms from a distance-to-death perspective. Psychol Aging. 2016;31:672-86.

24. Holvast $F$, Burger $H$, de Waal MM, et al. Loneliness is associated with poor prognosis in late-life depression: longitudinal analysis of the $\mathrm{Ne}$ therlands study of depression in older persons. J Affect Dis. 2015;185:1-7.

25. Cruwys T, Dingle GA, Haslam C, et al. Social group memberships protect against future depression, alleviate depression symptoms and prevent depression relapse. Social Sci Med. 2013;98:179-86.

26. Glei DA, Goldman N, Liu IW, et al. Sex differences in trajectories of depressive symptoms among older Taiwanese: the contribution of selected stressors and social factors. Aging Mental Health. 2013;17:773-83.

27. Montagnier D, Dartigues JF, Rouillon F, et al. Ageing and trajectories of depressive symptoms in community-dwelling men and women. Int $\mathrm{J}$ Geriatr Psychiatry. 2014;29:720-9.

28. Hudson E, Barrett A. Peer groups, employment status and depressive symptoms among older adults in Ireland. Journal of Population Ageing. 2014;7:43-54

29. Jang SN, Cho SI, Chang J, et al. Employment status and depressive symptoms in Koreans: results from a baseline survey of the Korean Longitudinal Study of Aging. J Gerontol B Psychol Sci Soc Sci. 2009;64:677-83

30. Chiao C, Weng LJ, Botticello AL. Economic strain and well-being in late life: findings from an 18-year population-based Longitudinal Study of older Taiwanese adults. J Public Health. 2012;34:217-27.

31. Huang $\mathrm{H}$, Russo J, Von Korff $M$, et al. The effect of changes in depressive symptoms on disability status in patients with diabetes. Psychosomatics. 2012;53:21-9.

32. Unutzer J, Katon W, Callahan CM, et al. Collaborative care management of late-life depression in the primary care setting: a randomized controlled trial. JAMA. 2002;288:2836-45.

33. Callahan CM, Kroenke K, Counsell SR, et al. Treatment of depression improves physical functioning in older adults. J Am Geriatr Soc. 2005:53:367-73.

34. Pérez-Zepeda MU, Arango-Lopera VE, Wagner FA, et al. Factors associated with help-seeking behaviors in Mexican older individuals with depressive symptoms: a cross-sectional study. Int J Geriatr Psychiatry. 2013;28:1260-9

35. Isaac V, Stewart R, Artero S, et al. Social activity and improvement in depressive symptoms in older people: a prospective community cohort study. Am J Geriatr Psychiatry. 2009;17:688-96.

36. Gutiérrez J, Rivera-Dommarco J, Shamah-Levy T, et al. Encuesta Nacional de Salud y Nutrición 2012. Resultados nacionales. Cuernavaca, México: Instituto Nacional de Salud Pública (MX); 2012.

37. Mejía-Arango S, Gutiérrez LM. Prevalence and incidence rates of dementia and cognitive impairment no dementia in the Mexican population: data from the Mexican Health and Aging Study. J Aging Health. 2011;23:1050-74

38. Wong R, Espinoza M, Palloni A. Adultos mayores mexicanos en contexto socioeconomico amplio: salud y envejecimiento. Salud Pública de Mexico. 2007;49(Suppl 4):S436-47.

39. Murphy RA, Hagaman AK, Reinders I, et al. Depressive trajectories and risk of disability and mortality in older adults: Iongitudinal findings from the Health, Aging, and Body Composition Study. J Gerontol A Biol Sci Med Sci. 2016 Feb;71(2):228-35. 